\title{
INFORMACIÓN DE LA ADMINISTRACIÓN GENERAL DEL ESTADO EN LA ERA DIGITAL: PUBLICACIONES OFICIALES
}

\section{Cristina Rodríguez-Vela}

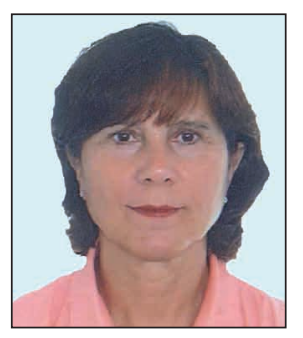

Cristina Rodríguez-Vela es licenciada en filosofía y letras por la Universidad Complutense de Madrid y diploma de estudios avanzados en la Facultad de Ciencias de la Información (especialidad de biblioteconomía y documentación) de la UCM con el trabajo de investigación "Las publicaciones oficiales: transparencia y accesibilidad". Su vida profesional se ha desarrollado en los campos de la documentación y de la edición pública. Ha dirigido proyectos sobre la implantación de sistemas de gestión de la documentación e información. Ha sido subdirectora general de publicaciones, documentación y archivo en el Ministerio de la Presidencia y actualmente desempeña la dirección del Departamento de Publicaciones y Fomento de la Investigación del Centro de Investigaciones Sociológicas (CIS). Es autora de diversos trabajos sobre lenguajes documentales, sistemas de gestión de la información y otros relativos a las publicaciones de la Administración General del Estado.

Centro de Investigaciones Sociológicas (CIS) Montalbán, 8. 28014 Madrid http://www.cis.es crvela@cis.es

\section{Resumen}

La Administración General del Estado (AGE) produce, difunde, conserva y reutiliza información para llevar a cabo la misión de servicio público que tiene encomendada. Utiliza las publicaciones, que han venido llamándose oficiales, para difundir la información relativa a las políticas públicas. Utilizamos el término publicación con el alcance que tiene en la era digital, que incluye cualquier recurso informativo en línea, incluidas las webs de los departamentos. La Administración reúne en dos importantes piezas la información sobre su propia actividad editora: el Catálogo de publicaciones de la AGE y la Guía de editores. La preservación y reutilización de la abundante y valiosa información del sector público son dos aspectos pendientes de organización. Las lagunas normativas que presentan en España estas dos facetas de actividad están actualmente en vías de solución.

\section{Palabras clave}

Administración General del Estado, Información del sector público, Difusión de la información, Preservación de la información, Reutilización de la información, Publicaciones oficiales, Servicio público, Catálogo.

\section{Title: Public administration information in the digital era: official publications}

\begin{abstract}
Governments produce, disseminate, preserve and reuse information in order to carry out the public services entrusted to them. They make use of the so-called official publications to disseminate information about public policies. We use the term publications in the sense that this word has attained in the digital era, that is, any digital resource online, including web pages from departments. The Spanish Administration provides two key elements of information about its publishing activity: the Catalogue of Publications of the General Administration of the State and the Guide to official publishers. The organizational developments for preserving and reuse the valuable and extensive information of the Spanish Public Sector are still pending, but the current regulatory gaps are in the process of being resolved.
\end{abstract}

\section{Keywords}

General Administration of the State, Public sector information, Dissemination of information, Preservation of information, Reuse of information, Official publications, Public service, Catalogue.

Rodríguez-Vela, Cristina. "Información de la Administración General del Estado en la era digital: publicaciones oficiales". El profesional de la información, 2011, mayo-junio, v. 20, n. 3, pp. 315-323. 


\section{Introducción}

Vamos a abordar este trabajo desde el punto de vista de la Administración como productora de información y, como actividad derivada, difusora de esa misma información. Excluimos de este análisis los boletines oficiales (diarios oficiales), que se han estudiado tradicionalmente en los manuales de fuentes de información como una de las más importantes publicaciones oficiales. Ciertamente son publicaciones de gran importancia, en cuanto permiten la publicidad de las normas, pero no son objeto de este trabajo puesto que el ámbito al que nos referimos viene definido por el Real decreto $118 / 2001$, y se refiere a aquellas que constituyen un soporte informativo y difusor de la actividad de la Administración.

Por lo que respecta al término información o documentación lo circunscribimos a las publicaciones de la Administración General del Estado (AGE), denominadas en la jerga profesional publicaciones oficiales.

Los términos administración e información se presentan con mucha frecuencia unidos, hasta el punto de que ha llegado a enunciarse que administración pública e información es lo mismo, lo que ha sido invocado por Muñoz-Cañavate (2001) al explicar que la Administración trabaja con información y el producto no es otro que información transformada.

La AGE difunde, conserva y reutiliza información en cumplimiento de la función de servicio público que tiene encomendada. Los aspectos de la conservación y reutilización son por el momento los más problemáticos si bien, afortunadamente, están en vías de solución en España a partir de normas que vienen a cubrir vacíos muy notables de carácter legal y organizativo.

La cultura digital transciende la actividad de la Administración, muy diversa, que necesariamente responde a las pautas de la sociedad de la información. El momento histórico por el que atravesamos, la implantación de la administración electrónica y la contribución de las TIC a los procedimientos ordinarios de la actividad, nos sitúa en un escenario tecnológico en el que el adjetivo digital o electrónico se supone por defecto.

Así pues, las publicaciones de la AGE tienden a presentarse, como no podía ser de otro modo, en versión electrónica. Pero aún más, dentro de los términos electrónico y digital, veremos que hay una creciente actividad de publicación en soporte no tangible. Esa es la información de fácil difusión que, sin embargo, por su naturaleza inestable, presenta más problemas a efectos de control y, por tanto, de preservación.

\section{Publicaciones oficiales y sociedad de la información}

\section{Las publicaciones oficiales como soporte informativo} y difusor de la actividad de la Administración

Estas publicaciones constituyen el soporte informativo y difusor de las actividades llevadas a cabo por la AGE, según el preámbulo del Real decreto 118/2001 de Ordenación de publicaciones oficiales, norma en la que se encuadra la actividad editora de la Administración.
La Administración utiliza las publicaciones para la difusión de sus políticas sectoriales, en cumplimiento de su misión de servicio público, con el objetivo de servir al ciudadano. Cuando se habla de la información del sector público, como término acuñado, hay una referencia desde una perspectiva general a toda aquella información que sirve al ciudadano (Muñoz-Cañavate, 2001).

La actividad editora de la Administración está ordenada mediante procedimientos bien definidos (Rodríguez-Vela, 2002) y con un decidido espíritu de transparencia. La organización y el sistema de gestión de la información actuales permiten a la Administración conseguir esa transparencia en sus actuaciones y en sus productos de información bibliográficos, sobre todo a partir del año 2000, como veremos más adelante. La AGE pone a disposición del ciudadano sus activos de información en línea, como corresponde al escenario cultural y tecnológico de la sociedad de la información.

\section{La actividad editora de la Administración se ordena mediante procedimientos bien definidos y con espíritu de transpa- rencia}

\section{La sociedad de la información}

La Administración realiza un esfuerzo en el desarrollo de su gestión electrónica, que ha recibido un impulso importante gracias a los diferentes planes del Gobierno y la aprobación de nueva legislación en 2007, de extraordinario impacto. La Ley 11/2007, de 22 de junio, de Acceso electrónico de los ciudadanos a los servicios públicos (Laecsp) subraya la relación con las administraciones públicas por medios electrónicos como un derecho de los ciudadanos y como una obligación correlativa para tales administraciones.

Además de ese enfoque centrado en el ciudadano, hay que tener en cuenta que el avance de la administración electrónica da respuesta a los compromisos comunitarios y a las iniciativas europeas puestas en marcha a partir del Consejo Europeo de Lisboa hasta la comunicación de la Comisión i2010: una sociedad de la información europea para el crecimiento y el empleo, en la que los servicios públicos en línea constituyen uno de los pilares básicos.

También en 2007 se publica la Ley 56/2007 de Medidas de impulso de la sociedad de la información. Esta última se enmarca en el Plan Avanza, aprobado por el Gobierno en 2005 , consistente en un conjunto de medidas para el período 2006-2010, con el propósito de extender el uso de las TIC para garantizar los derechos de los ciudadanos.

Vencido el plazo previsto por la iniciativa i2010, la Comisión Europea abrió un nuevo período con la aprobación en mayo de 2010 de la Agenda digital europea: estrategia 2020, para una economía digital próspera en el horizonte de otros diez años, que define acciones y políticas necesarias para maximizar los beneficios de la revolución digital.

En este contexto de cultura digital, de información en línea, de prestaciones al ciudadano y de puesta en valor de los 
beneficios de internet, se inscribe la actividad editora de la Administración como productora y difusora de información. Como productora analizaremos la creciente producción digital y el crecimiento de las publicaciones electrónicas. Como difusora dedicaremos un apartado especial a dos productos de especial importancia para el estudio y comprensión de las publicaciones oficiales: el Catálogo general de publicaciones de la AGE y la Guía de editores.

La organización y el sistema de gestión de la información permiten a la Administración conseguir la transparencia en sus actuaciones

\section{Las publicaciones electrónicas}

Hay una nueva acepción del término publicación, debido a la posibilidad de la publicación en internet (Cordón-García, 2004) que supone una nueva forma de comunicación y abre un interrogante en el control bibliográfico y, por tanto, en la preservación de las publicaciones digitales.

La revolución tecnológica de los soportes tiene un ritmo tan rápido que los textos legislativos relativos a la información o publicación buscan fórmulas válidas para el futuro. Ya en 2007, la Ley 10/2007 de la lectura, del libro y de las bibliotecas insiste en los soportes e incluso hace una previsión de futuro en una declaración abierta de lo que pueda ocurrir en relación con el mundo del libro, definiendo libro como toda "obra científica, artística, literaria o de cualquier otra índole que constituye una publicación unitaria en uno o varios volúmenes y que puede aparecer impresa o en cualquier otro soporte susceptible de lectura".

Los datos de la Memoria de publicaciones oficiales de la AGE 2009, última memoria disponible, son indicativos de la situación y manifiestan la incorporación de la Administración a esta revolución de la edición digital. De este modo, y sin entrar en un análisis minucioso de tipos de soportes, ni de formatos de edición electrónica, ni si añaden valor en su forma de publicación respecto a la información original (Bustos-Pretel, 2010), tomando el término electrónico en sentido amplio, la producción electrónica supuso en 2009

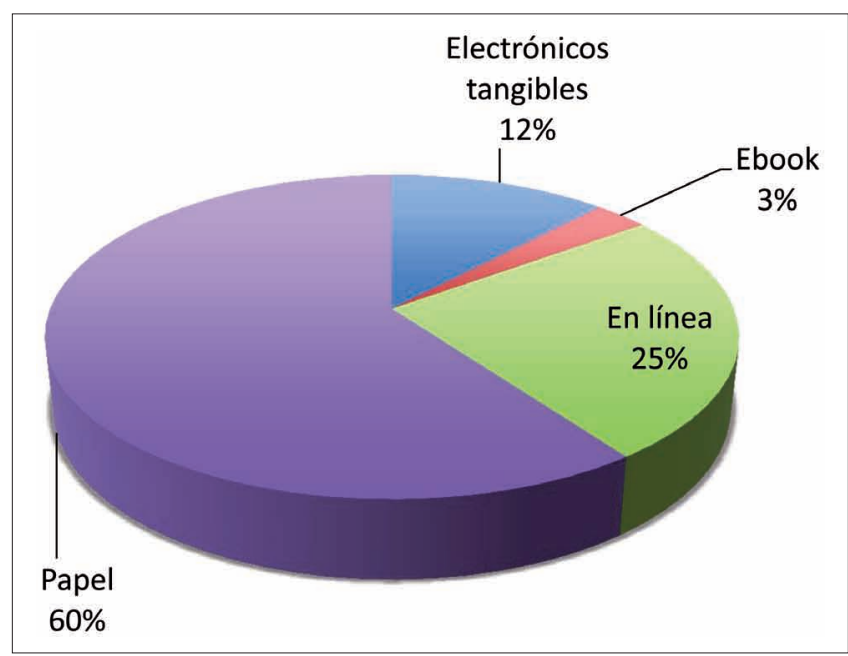

Producción por formatos de edición un $40 \%$ del total, lo que se traduce en un $60 \%$ para el soporte tradicional papel.

Interesa destacar que dentro del conjunto de obras electrónicas, el 69,7\% corresponde a publicaciones en línea, es decir, lo que se denomina obra o documentación intangible. Gran parte corresponde a publicaciones periódicas y bases de datos, y su carácter intangible e inestable nos traslada a los problemas que tiene este tipo de información a efectos de control bibliográfico y preservación (Rodríguez-Vela, 2008).

\section{Fuentes de información para las publicaciones de la AGE}

\subsection{Catálogo de publicaciones de la AGE (CPAGE)}

La creación del CPAGE es la respuesta informativa a la demanda creciente de transparencia por parte de la sociedad hacia la Administración. La histórica opacidad de conocimiento de las publicaciones oficiales se viene abajo con este instrumento de información, consultable en línea.

Hasta el momento, el Catálogo sigue siendo el elemento más importante del conjunto de ofertas informativas y de recuperación de la información que se ofrece al usuario (García-Ortiz; Moscoso, 2008).

El CPAGE es la respuesta informativa a la demanda creciente de transparencia por parte de la sociedad hacia la Administración

\section{Orígenes del proyecto CGPO}

El primitivo Catálogo de publicaciones oficiales, obra de la SG de Publicaciones Oficiales, tuvo una corta vida. Fue sustituido por un nuevo Catálogo general de publicaciones oficiales (CGPO), fruto del trabajo cooperativo de todos los departamentos, que se llevó a cabo entre 2002 y 2003, en torno a la idea de activar el comercio electrónico.

A grandes rasgos, los actores fueron el Ministerio de $\mathrm{Ha}$ cienda, que impulsó el proyecto, participó en el grupo de trabajo pero no entró en las fases operativas, que quedaron en manos de los Ministerios de Administraciones Públicas y Presidencia. Éstos se encargaban también de tareas complementarias tal y como queda establecido en un Protocolo de Coordinación firmado el 29 de mayo de 2003. Se trataba de un sistema que exigía la carga de datos mediante ficheros $\mathrm{xml}$ por parte de cada unidad editora, con la coordinación de la Subdirección de Publicaciones, Documentación y Archivo, en funciones de Secretaría de la Junta de Coordinación de Publicaciones Oficiales. El CGPO se puso en marcha en 2005 , con 15.000 registros bibliográficos. Sin embargo, hubo problemas con la estabilidad de la aplicación, y luego con la alimentación de datos, y quedó suspendida en octubre del 2006.

\section{Un nuevo catálogo}

Entretanto, y en paralelo, la SG de Publicaciones, Documentación y Archivo del Ministerio de la Presidencia había desa- 
rrollado Sicopo, un sistema de gestión para la coordinación de las publicaciones oficiales que incorporaba un procedimiento de trabajo en línea con conexión web (RodríguezVela; Ruiz-Esteban, 2008). Sicopo implicó que la introducción de datos de publicaciones se realizara directamente por las unidades editoras, y esa SG, en funciones de Secretaría de la Junta de Coordinación de Publicaciones Oficiales, revisa y consolida los datos- tanto bibliográficos como de proceso- siempre en el mismo sistema.

La estructura organizativa original tenía un diseño radial que suponía la autonomía de todas las unidades editoras a la hora de enviar los datos de sus publicaciones. Esta estructura demostró ser poco eficaz: la diversificación de tareas entre distintas unidades editoras producía inconsistencias y ralentización de algunas tareas de coordinación así como de la carga de datos.

A partir del segundo semestre de 2008 se puso en marcha una nueva versión del Catálogo general. Se estableció un plan de evaluación y relanzamiento del proyecto, cuyo desarrollo técnico iba a ser dependiente de la información enviada por Sicopo.

Como resultado de este proceso, y con la colaboración de la Secretaría de Estado para la Función Pública (DG para el Impulso de la Administración Electrónica y la SG de Innovación y Programas de Atención al Ciudadano), se comenzó un desarrollo técnico en el que la estructura de datos y definición de especificaciones del Catálogo general quedó a cargo de Ministerio de la Presidencia, y el desarrollo técnico a cargo del Ministerio de Administraciones Públicas, al que le correspondía su inclusión en el Portal 060.

La alimentación del Catálogo general se estableció mediante una estructura de datos exportados desde Sicopo, utilizando ficheros $\mathrm{xml}$ automatizados, y las especificaciones se orientaron a la facilidad de uso por parte del ciudadano. Esta nueva versión recibe nueva denominación: Catálogo de publicaciones de la $A G E$.

\section{Nueva etapa. El CPAGE se aloja en el servidor del BOE}

La Agencia Estatal BOE (Boletín Oficial del Estado) tiene encomendada, según su estatuto y de acuerdo con el contrato de gestión vigente, la distribución y venta de las publicaciones de la AGE. Además, mantiene una tienda electrónica que permite la compra en internet.

Teniendo en cuenta que:

- el $B O E$ es la única entidad capacitada para mantener la logística que implica la venta electrónica, y

- la SG de Publicaciones, Documentación y Archivo es la única que puede mantener la información sobre publicaciones oficiales,

se llega al acuerdo de alojar el Catálogo en el servidor del $B O E$. Se destaca la necesidad de mantener la titularidad del proyecto y de los contenidos del Catálogo general de la Junta de Coordinación de Publicaciones Oficiales.

Así se establece una gestión compartida que implica al $B O E$, como gestor en términos informáticos, y a la SG de Publi- caciones, Documentación y Archivo, como coordinadora en términos de contenido técnico.

Con esta actuación conjunta se alcanza un doble objetivo de transparencia y eficacia, se difunde la información y se posibilita la adquisición de la publicación mediante comercio electrónico, origen y objetivo del proyecto (Pérez-Rosado, 2004).

En el CPAGE se establece una gestión compartida que implica al $B O E$ y a la Subdirección General de Publicaciones, Documentación y Archivo

La nueva versión, que se puso en explotación y acceso público en el mes de septiembre de 2010, contiene un total de 43.500 registros bibliográficos, correspondientes a las múltiples unidades editoras.

Este Catálogo, que reúne la producción de toda la AGE desde al menos los últimos 10 años, y que permite al ciudadano la adquisición de obras procedentes de diferentes ministerios u organismos públicos, constituye un punto de información centralizado. Por otra parte, las obras de los diferentes ministerios se pueden consultar igualmente en los catálogos departamentales y adquirir en muchas de las librerías o puntos de venta de cada ministerio u organismo.

La estructura en campos responde a esquemas clásicos. Si bien se ha dado una especial importancia a los soportes en que se presentan las obras, con vistas a destacar el soporte electrónico y sobre todo la versión en línea. Desde el opac se puede acceder a la información digital. Estos materiales contenidos en el catálogo serán la base del Repositorio de publicaciones oficiales digitales.

\subsection{Guía de editores}

La otra fuente de información sobre publicaciones oficiales es la Guía de editores. Es la relación de todos los centros de publicaciones que tienen competencias para editar, además de todos los organismos públicos, ya sean organismos autónomos o agencias estatales. Reúne el conjunto de unidades editoras, actualmente 84, lo que facilita el acceso a una información originalmente dispersa.

\section{Cada registro de la Guía de editores es una radiografía de la unidad editora, tanto en lo referente a sus responsables como a sus servicios y su producción}

La Guía resume la diversidad de fuentes de producción y distribución, además de dar información directa sobre datos de interés para ciudadanos interesados y, especialmente, de sectores profesionales del libro.

Este repertorio de información, consultable en línea, mantiene la información permanentemente actualizada, aunque hasta el momento también se edita anualmente impreso y en cd-rom. 


GOBIERNO MINISTERIO
DE LA PRESIDENCIA

Ejemplo del Catálogo de publicaciones de la AGE, http://publicacionesoficiales.boe.es
En el ámbito de la AGE, el NIPO (orden ministerial de 30 de diciembre de 1993) es el identificador específico de las publicaciones oficiales, que se asigna a una obra junto a los otros identificadores internacionales (ISBN e ISSN) y al depósito legal (DL). La referencia de autoridad que ha ejercido el ISBN en la asignación de NIPO a las publicaciones unitarias explica que, en un principio, las bases de datos producidas por la Administración no tuvieran NIPO ya que estaban expresamente excluidas de los materiales a los que se asignaba ISBN. De igual modo estaban excluidas las webs por su carácter inestable. Sin embargo el ISBN se diferencia del NIPO en el tratamiento de las obras gratuitas. Su exclusión en estas obras ( $\mathrm{Ma}$ nual ISBN, 2007) queda recogida en el anexo de la Ley 10/2007 De la lectura, del libro y de las bibliotecas, lo que introduce más distancia en la pretendida

Cada registro de la Guía de editores es una radiografía de la unidad editora, tanto en lo referente a sus responsables como a sus servicios y su producción. La actividad editora de cada unidad queda reflejada. El NIPO (número de identificación de las publicaciones oficiales) como identificador encabeza cada registro, que informa además de la dirección electrónica y web de cada unidad editora.

La Guía de editores proporciona una descripción exhaustiva, que aporta abundante información susceptible de ser reelaborada, tratada o analizada para ulteriores trabajos de investigación.

\section{Conservación y reutilización de la información de la AGE}

Tanto la conservación del patrimonio documental o bibliográfico como la reutilización de la información del sector público han sido objeto de estudio por organizaciones internacionales, y se ha publicado normativa para orientar la actividad de la Administración en estas dos grandes áreas de actividad relacionadas con la información digital.

\subsection{Control bibliográfico y conservación de la infor- mación digital}

Los estudios sobre documentación y edición electrónicas señalan problemas como la inestabilidad y el control bibliográfico, que es en definitiva hablar de la conservación de esa información digital. La publicación en internet ha sobrepasado las posibilidades de control bibliográfico, lo que supone una laguna importante en las organizaciones productoras y difusoras de información digital.

La actualización continua y la volatilidad de los contenidos suponen una dificultad de control especial en los recursos electrónicos continuados (sobre todo para las bases de datos y sitios web). Según las Directrices de la Unesco, se estima que la vida media de las páginas de internet oscila entre cuarenta y cuatro días y dos años (Orera-Orera, 2008). semejanza entre NIPO e ISBN.

Hay que recordar que el NIPO no discrimina entre los diferentes tipos de obras. Su asignación afecta a toda la producción, lo que le asemeja al DL, que, naciendo con voluntad de control, es el principal medio para asegurar la preservación bibliográfica.

Pero el DL no satisfacía las necesidades de control de los nuevos documentos digitales, sobre todo de aquellos de naturaleza inestable. En España la normativa sobre DL data de los años 1957, 1971 y 1973, lo que nos sitúa en un contexto tecnológico preelectrónico (Cordón-García; Alonso-Arévalo; Martín-Rodero, 2010).

La preservación es una función primordial que tiene encomendada la Administración en beneficio de las generaciones tanto presentes como futuras. Esta preservación implica el acceso libre a la información de obras de dominio público al tiempo que protege los derechos de autor en el ámbito de la propiedad intelectual.

En el ámbito internacional, la Unesco ha auspiciado estudios para abordar la conservación del patrimonio cultural, que son una referencia para los estudiosos del tema (Orera-Orera, 2008; García-Melero, 2004; Agenjo; Hernández, 2008). Por su parte la Unión Europea ha sido muy activa en el estudio y recomendaciones para resolver la recogida de los materiales documentales en línea con fines de conservación y difusión.

Tanto para la conservación como para el intercambio de textos electrónicos son indispensables los metadatos que permiten organizar conjuntos de información de recursos digitales (Agenjo, 2005).

\section{El anteproyecto de ley sobre depósito legal}

Hasta ahora, en España, esa misión de conservación del patrimonio cultural y bibliográfico ha venido instrumentándose mediante la normativa vigente, claramente obsoleta. En la actualidad se encuentra en tramitación el Proyecto de ley 
de depósito legal, adaptado al momento tecnológico digital. El nuevo texto aborda los diferentes supuestos que se puedan dar en el mundo de la las publicaciones y la documentación digitales, en definitiva de la información digital. La verdadera importancia de esta norma, tan esperada, que viene a cubrir un vacío legal, es que contempla el depósito de los nuevos soportes de la edición y de los documentos en red.

La nueva ley de DL tendrá en cuenta la información digital

El primero de los veinte artículos del anteproyecto contiene las definiciones de una amplia relación de términos propios de la cultura digital y sociedad de la información, que van a ser un punto de referencia obligado para la literatura especializada.

Es interesante el esquema organizativo de la actividad que configura la norma, basado en el Estado de las autonomías y concebida como actividad descentralizada. Por otra parte, hace una previsión de la organización de centros depositarios y centros de conservación con un papel relevante para la Biblioteca Nacional que ejercerá, además, la función de seguimiento de la normativa.

Por lo que respecta a las publicaciones oficiales, estarán representadas en un órgano de carácter consultivo, de participación del sector, en el que tendrán voz a través de un miembro de la Junta de Coordinación de Publicaciones Oficiales (disposición adicional segunda).

Así pues, finalizada la tramitación, tendremos una Ley que vendrá a ocuparse de aquellos materiales que escapaban al control bibliográfico, ya que define como objeto de depósito legal "todo tipo de publicaciones producidas en España, por cualquier procedimiento de producción y distribuidas en cualquier soporte, tangible o intangible" (art. 4.1) y enumera los tipos de publicaciones y recursos (art. 4.3), aludiendo a: "recursos continuados", "recursos integrables como las hojas sueltas actualizables", "sitios web cuyo contenido pueda variar con el tiempo", etc.

Las dos novedades más relevantes de este proyecto de ley son: la obligación de constituir el depósito pasa del impresor al editor, y el archivo de las publicaciones electrónicas en línea, si bien hace una previsión de un año para la constitución del procedimiento de DL de estas publicaciones electrónicas (disposición adicional tercera).

Las dos novedades del nuevo DL son: la obligación de constituir el depósito pasa del impresor al editor, y la conservación de las publicaciones electrónicas en línea

Con la entrada en vigor de esta ley se garantizará el depósito de esos materiales intangibles, de modo que los repositorios de publicaciones digitales tendrán el apoyo normativo que necesitaban para su constitución. El viejo proyecto de
Repositorio de publicaciones oficiales recibirá nuevo impulso con el desarrollo de la nueva normativa.

\subsection{Reutilización de la información del sector público (RISP)}

La Ley 37/2007, de 16 de noviembre, sobre Reutilización de la información de sector público, viene a reforzar el concepto de transparencia de la actuación de la Administración y establece el régimen jurídico para la reutilización de dicha información. Por medio de esta Ley se incorpora a nuestro ordenamiento jurídico la Directiva 2003/98/CE de 17 de noviembre de 2003 del Parlamento Europeo y del Consejo relativa a la Reutilización de la información del sector público.

Según dice el preámbulo, "las administraciones y organismos recogen, producen, reproducen y difunden documentos para llevar a cabo la misión de servicio público que tienen encomendada. La utilización de dichos documentos por otros motivos ya sea con fines comerciales o no comerciales constituye una reutilización".

Se trata de la explotación de la información en el sector público (ISP) de numerosos campos de interés como información social, económica, jurídica, geográfica, meteorológica, turística, sobre empresas, patentes, educación, etc.

EI RD de desarrollo de la Ley de RISP trata temas organizativos e instrumentales, básicos para la promoción de la reutilización de la información

Lo que realmente es importante en esta norma es el concepto de reutilización vinculado al valor añadido y la apuesta por el crecimiento del sector de contenidos digitales. Este es el punto neurálgico que parte de un supuesto implícito: todos los servicios se valdrán de medios electrónicos, como corresponde a la sociedad de la información. Este contexto conceptual nos aproxima al mundo de las publicaciones oficiales por su definición y funciones como vehículos de información sobre la actividad de la Administración.

Las publicaciones oficiales contienen información primaria (como las publicaciones estadísticas o las cartográficas), o secundaria elaborada a partir de la primaria. La reutilización es un segundo nivel de tratamiento de la información generada desde las instancias públicas y tiene una connotación económica, por lo que la ley habla del "mercado de la reutilización de la ISP".

La actividad editora de la Administración y los organismos públicos, siempre sustentada por las TIC, desborda la acepción tradicional para ofrecer nuevos servicios y productos de valor añadido, una amplia gama de contenidos digitales.

\section{El desarrollo reglamentario}

Se hará mediante real decreto, que ha estado en fase de consulta pública, y que se encuentra en tramitación. Se enmarca en el conjunto de medidas que constituyen la Estrategia 2011-2015 del Plan Avanza 2. Dentro de este Plan se encuentra igualmente el Proyecto Aporta, que entraña una 
iniciativa en apoyo de la Ley 37/2007. Este proyecto pretende impulsar el mercado y fomentar la cultura de la reutilización de la información del sector público (RISP) y facilitar la puesta a disposición de la ISP por parte de la Administración.

En grandes líneas, el real decreto señalará ciertas obligaciones: se autoriza la reutilización siempre que el acceso no esté restringido; las administraciones informarán sobre los documentos reutilizables; se definen responsables de cada departamento ministerial u organismo; se regulan los mecanismos de coordinación; se apoya normativamente el catálogo de información pública reutilizable, creado inicialmente en el marco del Proyecto Aporta; y se establecen obligaciones de actualización del catálogo por parte de las administraciones y los organismos.

La regla general será la puesta a disposición de la información sin sujeción a condiciones específicas, no obstante éstas se marcarán cuando sean necesarias y se precisará la regulación de los documentos reutilizables sujetos a propiedad intelectual o que contengan datos personales.

Este real decreto apunta un diseño de organización estableciendo un responsable en cada departamento en materia de reutilización de la ISP y concreta la adscripción de ese órgano a las subsecretarías de los departamentos o secretarías generales de los organismos dependientes. Igualmente explicita la necesidad de coordinar la información administrativa del departamento y la elaboración de un informe anual que será publicado en su sede electrónica. Como se deduce del articulado, el real decreto trata temas organizativos e instrumentales de importancia básica para el propósito de promoción de la reutilización de la información.

Es importante destacar la previsión que hace este reglamento de la organización a efectos de mantener la información estructurada con metadatos, indicando fecha de actualización y organizada en bases de datos. No en vano el término metadato, utilizado desde mediados de los años noventa coincidiendo con la explosión de los recursos electrónicos en la web y de la misma web, venía a ser sinónimo de descripción de recursos electrónicos (Ajenjo; Hernández, 2009).

Pero si hay que destacar algo de especial importancia en esta nueva norma, es el Catálogo de recursos de información pública reutilizable, quizá la pieza clave en la instrumentación de este servicio, que constituye un único punto de acceso a los distintos recursos.

Desde el punto de vista del análisis de los instrumentos que posibiliten el logro de un buen sistema habría que abordar con más detalle los aspectos organizativos:

- cómo los departamentos van a mantener ese Catálogo de recursos de información pública, que hemos denominado pieza clave unificadora de la información, y qué órgano centralizará la información;

- cómo se va a estructurar la información y se van a definir los procedimientos que afectan transversalmente la recogida de información. Deberá corresponder como tarea especializada a un órgano concreto, que sea responsable del tratamiento, gestión y suministro de la información reutilizable.
Esta actividad de la Administración exige la concurrencia de muchos agentes especializados porque, parafraseando al profesor Ramos, "la reutilización es un proceso multifacético en el que concurren aspectos jurídicos, económicos, informativos-documentales, comerciales, de sistemas de información, etc." (Ramos-Simón, 2008).

EI RD de desarrollo de la Ley de RISP trata temas organizativos e instrumentales, de importancia básica para la reutilización de la información

A la luz de este análisis, es inevitable, por analogía, pensar en la estructura organizativa de la actividad editora de la Administración. Parece razonable contar en esta actividad de reutilización de la AGE con estructuras administrativas consolidadas y con modelos de organización bien implantados, como es el caso de la articulación de la actividad editora de la AGE.

\section{Conclusiones}

Después de diferentes versiones el Catálogo de publicaciones de la AGE y la Guía de editores son las piezas clave de las que se vale la AGE para la difusión de la actividad productora de información. La nueva versión del primero supone una indudable mejora de la actividad de difusión de la información. Con su alojamiento en el servidor del $B O E$, se da cumplimiento a la difusión de la información bibliográfica y al mismo tiempo al proyecto de comercio electrónico, sustentado en la librería del $B O E$.

Respecto a la conservación de la producción documental digital, la Ley de depósito legal (todavía proyecto de ley) vendrá a cubrir el vacío existente sobre la conservación de los nuevos soportes de la edición y de los documentos en red. Los recursos digitales y los materiales informativos intangibles serán objeto de conservación. Con esta nueva ley se asegurará la constitución de repositorios de publicaciones digitales, y por lo que se refiere a publicaciones electrónicas oficiales, repercutirá en la constitución del Repositorio de publicaciones oficiales, viejo proyecto pendiente de acometer.

Por lo que respecta a la reutilización, el Reglamento de desarrollo de la Ley 37/2007 de Reutilización de la información del sector público establece las obligaciones para la Administración y apunta órganos responsables para llevar a cabo esta actividad, si bien será necesario un desarrollo más detallado que contribuya a la formación del Catálogo de recursos de información pública, pieza clave en la actividad de reutilización.

La coordinación tan necesaria en esta actividad y el Catálogo unificado de información reutilizable, fruto del trabajo de cooperación entre los diferentes departamentos, nos traslada por analogía a la actividad editorial y concretamente al órgano colegiado interministerial que ejerce la coordinación editorial: la Junta de Coordinación de Publicaciones Oficiales.

Partiendo de que las soluciones tecnológicas apoyadas en estructuras organizativas son condiciones indispensables 
para garantizar el éxito de la actividad, podemos concluir que las dos actividades analizadas -las de preservación y de reutilización de la información de la AGE-, que se presentaban en la introducción como problemáticas, están en vías de solución, puesto que sus respectivos desarrollos normativos están en estos momentos en fase de tramitación y abordan los tan necesarios aspectos organizativos.

\section{Nota}

El Anteproyecto de Ley de depósito legal fue aprobado en el Consejo de Ministros el 18 de marzo de 2011.

\section{Bibliografía utilizada}

Agenjo, Xavier; Hernández, Francisca. "Exegi monumentum aere perennius (Hor. C. 3.30): preservación a largo plazo de materiales bibliográficos digitalizados". Boletín de la Anabad, t. LVIII, n. 2, 2008, pp. 101-111.

http://eprints.rclis.org/handle/10760/14365

Agenjo, Xavier; Hernández, Francisca. "La biblioteca virtual: función y planteamiento". Boletín de la Anabad, v. LIX, n. 4, 2009, pp. 25-105.

http://eprints.rclis.org/bitstream/10760/14352/1/La_bi blioteca_virtual_final.pdf

Barrueco, José-Manuel; Cordón-García, José-Antonio. “Revistas electrónicas y normalización". Revista general de información y documentación, 1997, v. 7, n. 2, pp. 365-374. http://revistas.ucm.es/byd/11321873/articulos/RGID 9797220365A.PDF

Bustos-Pretel, Gerardo. "La Administración tras la meta de las publicaciones electrónicas de calidad". Delibros, diciembre 2010.

Catálogo de publicaciones de la AGE

http://publicacionesoficiales.boe.es

Comisión de las Comunidades Europeas. eEurope 2005: una sociedad de la información para todos: plan de acción que se presentará con vistas al Consejo Europeo de Sevilla, 21-22 junio de 2002. COM (2002) 0263 final, 2002.

Comisión de las Comunidades Europeas. Hacia la Europa basada en el conocimiento: la Unión Europea y la sociedad de la información. Luxemburgo: Oficina de Publicaciones Oficiales de las Comunidades Europeas, 2003.

Comisión de las Comunidades Europeas. Plan de acción sobre administración electrónica i2010: acelerar la administración electrónica en Europa en beneficio de todos. SEC(2006) 511. $\operatorname{COM}(2006) 173$ final, 2006.

Comisión de las Comunidades Europeas. Iniciativa europea i2010 para la inclusión digital: participar en la sociedad de la información. SEC(2007)1469, SEC(2007)1470. COM(2007) 694 final, 2007.

Comisión de las Comunidades Europeas. Europe 2020: une stratégie pour une croissance intelligente, durable et inclusive. COM (2010) 2020. Bruselas, 2010.

Commission Française pour I'Unesco. La société de l'information: glossaire critique. París: La Documentation Française, 2005.
Cordón-García, José-Antonio. "El depósito legal y los recursos digitales en línea". En: I Jornadas sobre bibliotecas nacionales: las bibliotecas nacionales del siglo XXI, 2006, pp. 97-114.

http://eprints.rclis.org/bitstream/10760/15036/1/Deposi to_legal_y_nuevas_tec..pdf

Cordón-García, José-Antonio. "Prólogo”. En: Alonso-Berrocal, José L.; Figuerola, Carlos G.; Zazo, Ángel F. Cibermetría: nuevas técnicas de estudio aplicables al Web. Gijón: Trea, 2004. ISBN: 8497041143.

Cordón-García, José-Antonio; Alonso-Arévalo, Julio; Martín-Rodero, Helena. "Los libros electrónicos: la tercera ola de la revolución digital”. Anales de documentación, 2010, v. 13, pp. 53-80.

http://revistas.um.es/analesdoc/article/download/ 106991/101681

García-Melero, Luis-Ángel. "Algunas iniciativas relacionadas con la recopilación y conservación del patrimonio digital". Boletín de la Anabad, 2004, v. 54, n. 1-2, pp. 429-456.

http://www.anabad.org/archivo/docdow.php?id=143

García-Melero, Luis-Ángel. "La biblioteca digital revisitada". Boletín de la Anabad, 2009, v. LIX, n. 4, pp. 123-146. http://eprints.rclis.org/bitstream/10760/13628/1/LA_BI BLIOTECA_DIGITAL_REVISITADA.pdf

García-Ortiz, Francisco M.; Moscoso, Purificación. “El portal bibliotecario y el opac: ayuda en línea específica y compartida". Boletín de la Anabad, 2008, n. 2, pp. 135-148.

Guía de editores

http://servicios.mpr.es/publicaciones/BuscadorGuiaEdito res.aspx

ISBN Users' manual. Berlín: International ISBN Agency, 2005. ISBN 3880531137.

Ley 10/2007, de 22 de junio, De la lectura, del libro y de las bibliotecas. BOE, 23 de junio de 2007, n. 150, p. 27140.

Ley 11/2007, de 22 de junio, de Acceso electrónico de los ciudadanos a los servicios públicos. BOE, 23 de junio de 2007, n. 150, p. 27150.

Ley 37/2007, de 16 de noviembre, sobre Reutilización de la información del sector público. BOE, 17 de noviembre de 2007, n. 276, p. 47160.

Ley 56/2007, de 28 de diciembre, de Medidas de impulso de la sociedad de la información. BOE, 29 de diciembre de 2007, n. 312, p.53701.

López-Yepes, José. "El desarrollo de los sistemas de información y documentación”. Revista general de información y documentación, 1991, v. 1, n. 2, pp. 23-34.

http://revistas.ucm.es/byd/11321873/articulos/RGID 9191220023A.PDF

Maciá, Mateo. "Publicaciones oficiales: el Diario oficial de las comunidades europeas (DOCE)". Revista de la administración pública, 1994, n. 133, enero-abril, pp. 543-560. http://www.cepc.es/rap/Publicaciones/Revistas/1/1994_ 133_543.PDF

Moreiro-González, José-Antonio. Conceptos introductorios 
al estudio de la información documental. Salvador (Bahía): Edufba, 2005. ISBN: 8523203532.

Moreiro-González, José-Antonio. "La industria de los contenidos". En: Caridad-Sebastián, Mercedes (Coord.). La sociedad de la información. Política, tecnología e industria de los contenidos. Madrid: Centro de Estudios Ramón Areces, Universidad Carlos III de Madrid, 1999, pp. 243-265. ISBN: 8480044063.

Muñoz-Cañavate, Antonio. "Una aproximación a la información del sector público: la información de las administraciones públicas". Revista general de información y documentación, 2001, v. 11, n. 1, pp. 33-47.

http://revistas.ucm.es/byd/11321873/articulos/RGID 0101120033A.PDF

Orden del Ministerio de la Presidencia de 30 de diciembre de 1993 por la que se Regula el número de identificación de las publicaciones oficiales. BOE, 31 de diciembre de 1993, n. 313, p. 37912.

Orera-Orera, Luisa. "Preservación digital y bibliotecas: un nuevo escenario". Revista general de información y documentación", n. 18, 2008, pp. 9-24.

http://revistas.ucm.es/byd/11321873/articulos/RGID 0808110009A.PDF

Pérez-Rosado, José M. et al. "El proyecto de comercio electrónico de publicaciones oficiales". En: Tecnimap 2004: VIII Jornadas sobre tecnologías de la información para la modernización de las administraciones públicas. Murcia, 29 de septiembre-1 de octubre 2004.

Plan Avanza 2

http://www.planavanza.es

Plan Estratégico de la Laecsp

http://www.060.es

Proyecto Aporta: reutilización de la información del sector público.

http://www.aporta.es

Ramos-Simón, L. Fernando (coord.). Bases de datos de libre acceso difundidas por la Administración General del Estado. Madrid: Editorial Complutense, Ministerio de Cultura, Facultad de Ciencias de la Documentación de la UCM, 2008. ISBN: 9788474919400.
Ramos-Simón, L. Fernando; Mendo-Carmona, Concepción; Arquero-Avilés, Rosario. "La producción informativa y documental del Estado: hacia un inventario de los recursos públicos". Revista española de documentación científica, 2009, v. 32, n. 1, pp. 40-59.

http://redc.revistas.csic.es/index.php/redc/article/down load/452/464

Ramos-Simón, L. Fernando et al. "Diseño de modelos para el análisis de la información en el sector público". En: Infogestión: gas Jornadas españolas de documentación, Fesabid, 2005, Madrid, 14-15 de abril. ISBN: 8493033553.

Real decreto 118/2001, de 9 de febrero, de Ordenación de Publicaciones Oficiales. BOE, 10 de diciembre de 2001, n. 36, p. 5163.

Rodríguez-Bravo, Blanca. "Revisión de las clasificaciones documentales basadas en el soporte". Revista española de documentación científica, 2002, v. 25, n. 1, pp. 74-86.

http://redc.revistas.csic.es/index.php/redc/article/view/ $87 / 149$

Rodríguez-Bravo, Blanca. "Los repositorios de información, guardianes de la memoria digital". Anales de documentación, 2007, n. 10, pp. 361-374.

http://revistas.um.es/analesdoc/article/viewFile/1251/ 1301

Rodríguez-Vela, Cristina. "Introducción". En: RodríguezVela, Cristina (coord.). Publicaciones periódicas oficiales. Madrid: Ministerio de la Presidencia, 2008, pp. 11-17. ISBN: 9788474711400.

Rodríguez-Vela, Cristina. "Simplificación de procedimientos en la gestión de las publicaciones oficiales". En: Tecnimap 2002: VII Jornadas sobre tecnologías de la información para la modernización de las administraciones públicas. A Coruña, 15-18 de octubre 2002.

Rodríguez-Vela, Cristina; Ruiz-Esteban, Yolanda. "Nueva organización de la información referida a publicaciones oficiales: el sistema de gestión Sicopo". En: Actas de las X Jornadas de gestión de la información: la dimensión del cambio: usuarios, servicios y profesionales. Madrid: Sedic, 2008, pp. 131-145.

Unesco; UIT. "Cumbre mundial sobre la sociedad de la información: plan de acción”. En: Cumbre mundial sobre la sociedad de la información: Ginebra 2003-Túnez 2005, 2004.

\section{Suscripción EPI sólo online}

Pensando sobre todo en los posibles suscriptores latinoamericanos, ya no es obligatorio pagar la suscripción impresa de EPI para acceder a la online.

EPI se ofrece a instituciones en suscripción "sólo online" a un precio considerablemente más reducido (90 euros/año), puesto que en esta modalidad no hay que cubrir los gastos de imprenta ni de correo postal. 